\title{
Virtual Process Engineering - An approach to integrate VR, FEM, and simulation tools in the manufacturing chain
}

\author{
Fritz Klocke and Andreas M. Straube ${ }^{a}$ \\ Fraunhofer Institute for Production Technology IPT, Department Process Technology, Steinbachstrasse 17, 52074 Aachen, \\ Germany
}

Received 30 June 2003, Accepted 20 October 2003

\begin{abstract}
Today's businesses continually search for ways to increase productivity. To achieve this goal, shorter product development cycles and shorter manufacturing times are required. In both cases, problems that may arise during development must be identified much faster. In product development, this is done using virtual prototypes that can be easily duplicated and shared between everyone involved. In addition to this product design process, the stability of the manufacturing process and the resulting product quality ultimately determine the product's profitability. Therefore the manufacturing concepts of a new product can best be demonstrated using Virtual Manufacturing. The subset of Virtual Manufacturing where material properties of the product and machine tools and the physics of the process are investigated is called Virtual Process Engineering. Virtual Process Engineering utilizes Finite Element Methods (FEM) and simulations for the computation of the manufacturing process and Virtual Reality (VR) for quick and intuitive examination of the computation results.
\end{abstract}

Key words: FEM simulation / manufacturing chain / Virtual Reality / interfaces / Virtual Process Engineering

\section{Introduction}

Every corporation seeks to increase its competitive position in the markets through the production and development of its products. Increasing competition, rising standards for quality, and shorter product development times demand companies to optimize their product development processes [1]. To continually adapt to this changing business environment requires innovative product development, successful sales, good service, and economic manufacturing methods. One way to achieve economic manufacturing is to employ Virtual Manufacturing. According to Nahavandi and Preece [2], Virtual Manufacturing can be described as a simulated model of the actual manufacturing setup which holds all the information relating to the process, the process control and management, and product specific data. A similar definition is given by Hitchcock et al. [3]. In summary, these definitions can be combined and expressed in the following way as stated by Shukla et al. [4]: "Virtual Manufacturing aims to integrate diverse manufacturing-related technologies under a common umbrella, using Virtual Reality technology".

\footnotetext{
${ }^{a}$ Corresponding author:

andreas.straube@ipt.fraunhofer.de
}

Virtual Manufacturing can be divided into three areas according to Mukundan [5]: product, process, and resources. The product domain covers the design of individual parts, including the data and data links throughout the product life cycle. The process domain addresses the detailed planning of the manufacturing process, and the resource domain simulates product assembly by creating a complete digital mock-up of the manufacturing plant. This paper concentrates on the process domain. According to Mukundan the process domain includes the assignment of resources, the optimisation of workflow, through the linking of multiple processes, the application of process simulation, and collision checking. Robertson and Perera name the primary benefits of Virtual Manufacturing to be decision management and visualisation [6]. According to Roll et al. [7] process simulation consists of all methods and procedures that allow for the approximate determination of magnitudes for the real process (chemical and physical) prior to implementation of the real process. In the area of metal cutting, this process simulation focuses on the description of chip formation, temperature, and force distribution, all of which seeks to improve tool life and workpiece quality [8]. In contrast to the process simulations described by Mukundan [5], this paper focuses 


\section{Nomenclature}

\begin{tabular}{|ll|}
\hline ASCII & American Standard Code for Information Interchange \\
CAD & Computer Aided Design \\
CAE & Computer Aided Engineering \\
CAx & Computer Aided x \\
CNC & Computer Numerical Control \\
DMU & digital mock-up \\
FEM & Finite Element Method \\
Fps & frames per second \\
LOD & level of detail \\
NC & numerical control \\
PALOD & performance adaptive level of detail \\
STEP & Standard for the Exchange of Product Model Data \\
STL & Standard Triangulation Language \\
VR & Virtual Reality \\
\hline
\end{tabular}

on process simulation described by Roll et al. [7] which is primarily based upon Finite Element Methods (FEM) and includes material science parameters in the models. This type of simulation will be referred to as Process Engineering from this point on. Process Engineering is highly cost-effective, because it allows experiments to be performed without the down time of expensive machine tools or necessity of building and optimising expensive tools used in conventional manufacturing methods. Furthermore, Process Engineering is faster than performing real experiments and provides more flexibility. A wide variety of simulated experiments can be performed, and additional parameters that cannot be measured in the real process can also be studied. Taking VR into account, we arrive at Virtual Process Engineering. As described by Cobb et al. [9], Virtual Reality plays an integrating role. In our research, it is used in the post processing of FEM simulations and in the combined visualisation of the results from different FEM programs. A second approach of integration is to exchange FEM data between simulations of two manufacturing processes where the first process alters the material properties of the workpiece and the second process in the FEM simulation receives and accounts for this change. The interest in Virtual Process Engineering is enormous as shown by an expression of interest submitted to the European Commission [10].

\section{The manufacturing process chain as part of the product life cycle}

Several concepts for a manufacturing process chain exist [11], and all share the idea that the manufacturing process chain is part of the product life cycle. The product life cycle is divided into product planning, development and construction, process planning, product manufacturing, sales and support, use and service, and disposal and recycling. In this context, the three topics of development and construction, process planning, and product manufacturing comprise the manufacturing process chain [11]. In the following it will be focused on the manufacturing process chain.

\section{CAx tools in the manufacturing process chain}

The first Computer Aided x (CAx) tool used in the development and construction phase of a product is Computer Aided Design, or CAD. The geometry of the product is defined including all materials, dimensions, and tolerances. Many small- and medium-sized companies still use 2D CAD. Depending on the complexity of the product, this may be sufficient. For products that are more complex or entire assemblies, 3D CAD is a more effective tool. With 3D software tools, simple collisions and intersections between parts can be easily identified during the design. As the next step, digital mock-ups (DMU) can be generated based on the 3D CAD data. These DMUs are used for the detection of constructional flaws and collisions in complex assemblies, simple assembly simulations, and animation of assembly parts. Computer Aided Engineering (CAE) in the form of Finite Element simulations is used to analyse stress within the product structure. These FEM simulations are normally linear because no plastic deformation is involved. Multi-body simulation is used for detailed analysis of the kinematic and dynamic behaviour of multi-body systems during all stages of the design.

During the process planning, FEM and constitutive equations are used to determine optimal manufacturing process parameters. These FEM simulations are mostly non-linear, such as in the forming process where plastic deformation of the workpiece material takes place. Many types of process simulations are described by Klocke et al. [12]. An optimised manufacturing process will lead to improved tool life and improved workpiece quality. Furthermore, these process simulations also focus on the investigation of new materials and manufacturing advancements. At this time, the properties of the tool and the machine tool are not considered. The tool is typically modelled as a rigid body, which means that, for example, a drill will not elastically deform during the manufacturing process. A rigid drill might be sufficient if the workpiece with material behaviour and subsurface effects is under investigation. If research focuses on the tool behaviour and the tool load a rigid tool is insufficient. 
One reason that the machine tool is ignored is that most of the modelling systems are specialised for the simulation of plastic or elastic deformation and not for both. Another reason is the large amount of computation time needed. Normally each FEM simulation has its own post processor and for that reason it is difficult to visualise the manufacturing process and the responses of the tool and the machine in one environment. In this situation, VR post processing provided by Covise/Cover [13], which has interfaces to different FEM programs, can help.

The simulation of numerical control (NC) is another application for CAx in the manufacturing chain. The accuracy of a NC program can be verified using a simulated $\mathrm{CNC}$ and a virtual representation of the machine tool and the workpiece. The numerical code is read step-bystep, and the virtual machine performs the corresponding movements. The virtual workpiece consequently changes its shape due to the machining process. Collisions between the tool and other parts are visualised using the virtual representations of machine tool and workpiece. In an additional window the active steps of the numerical code are shown and the step which causes a collision is highlighted. This kind of software is provided by CGTech.

\section{Extending the paradigm of Virtual Reality}

There are several VR applications in the field of development and construction. The Fraunhofer IPT is using a Virtual Reality Lab that consists of an L-shaped twosided walk-in active stereo projection system with electromagnetic tracking, a Silicon Graphics Onyx 3200 with two graphics pipes, and several VR software packages, some of which will be described in the following. First, the Design Review will be focused on. The CAD data is converted into a triangulated format and visualised in a virtual 3D environment. At the Fraunhofer IPT, a combined version of Design Review and Digital MockUp (DMU) is used to analyse design of machine tools. For example, a machine tool for processing silicon wafers is constructed with the CAD system Solid Designer. Using the STEP-interface (Standard for the Exchange of Product Model Data) the model is exported and then imported into the CAD system Pro Engineer, which has a direct interface to the VR program DivisionMockup. The CAD data is not reduced prior to its transfer from Pro Engineer and thus results in a very detailed VR model consisting of 435000 polygons. This huge data set can be handled using a performance adaptive level of detail (PALOD) technique, which adjusts the model complexity to achieve a desired frame rate. If the acquired frame rate decreases the geometries are automatically switched to a lower LOD so that the desired frame rate is restored. Using this technique, it is possible to achieve a constant frame rate of $10 \mathrm{fps}$ on the Silicon Graphics Onyx 3200 with two pipes for the model of a silicon-wafer grinding machine. In this case the model is reduced from 435000 to 87000 polygons using PALOD without negative effects on the visual appearance. A virtual menu has been developed that provides the ability to rotate the entire machine, to hide and show parts of the machine tool and to display the results of a FEM simulation on a virtual monitor (see Fig. 1). These three aspects are elements of a Design Review scenario. The DMU of the machine is evaluated by animating machine tool components, which are checked for collisions against each other. The tool carriage axes in the $x, y$ and $z$ directions can be grasped in the virtual environment with a virtual hand and moved within their constructional limits. Additionally, the front and back of the machine tool can be opened. The CAD data exposes a collision between the tool carriage in the $y$-axis and a static coverage plate, a problem discovered by creating and testing the DMU.

With the VR system virtual design 2 [14], an assembly simulation is performed in the virtual environment. The grinding tool of the wafer-grinding machine is mounted into a support. The tool is selected with a virtual laser beam. A procedure is activated, which then starts displaying an assembly path. Now the tool is selected with a virtual hand. Two options for collision detection can be chosen. With the first choice, a "ghost" will appear instead of the object when the object collides with some other impenetrable part. The object itself will remain at the last valid (or collision-free) position while the ghost continues to move, thus showing the "real" position of the object as if there had been no collision. As soon as the collision is resolved or the ghost is moved to a collision-free position, the ghost will disappear, and the object itself will begin to move again [14]. This option allows for the rapid check of an assembly. Joining two parts, such as in a flange, is not possible this way however. In the second choice, collision detection in a so-called "glideable" mode is possible. With this method, the flange can be joined with the support. Only the necessary components within the scene are visible in this case. Training of the user on the assembly simulation in the virtual environment is needed because in the glideable mode, graphics performance decreases. With a scene of 155000 polygons, a frame rate of $7 \mathrm{fps}$ has been achieved.

In the research project "ProViT - Digital Product Development with virtually cooperating teams using optical networks" the Fraunhofer IPT is working in conjunction with the Fraunhofer IMK on a scenario for a Design Review and an assembly simulation in a shared virtual environment. The wafer-grinding machine requires air pressure and water to clean the silicon wafer before and after processing. As a virtual prototype, it is difficult to determine the path of tubes and their lengths. Efforts to solve this problem have yielded a realtime simulation of an elastic tube using FEM and precomputed Green's functions [15]. With this simulation, it is possible to lay a tube within the machine tool, to fix it at certain positions, and to cut it at the end. The total length is displayed on the tube-laying device. The virtual tubes possess real material parameters and properties. The aim of the ProViT project is to combine Virtual Reality and video conferencing to support a collaborative product development. This means that your project partners will be able to stand inside their VR system and be filmed with cameras. 


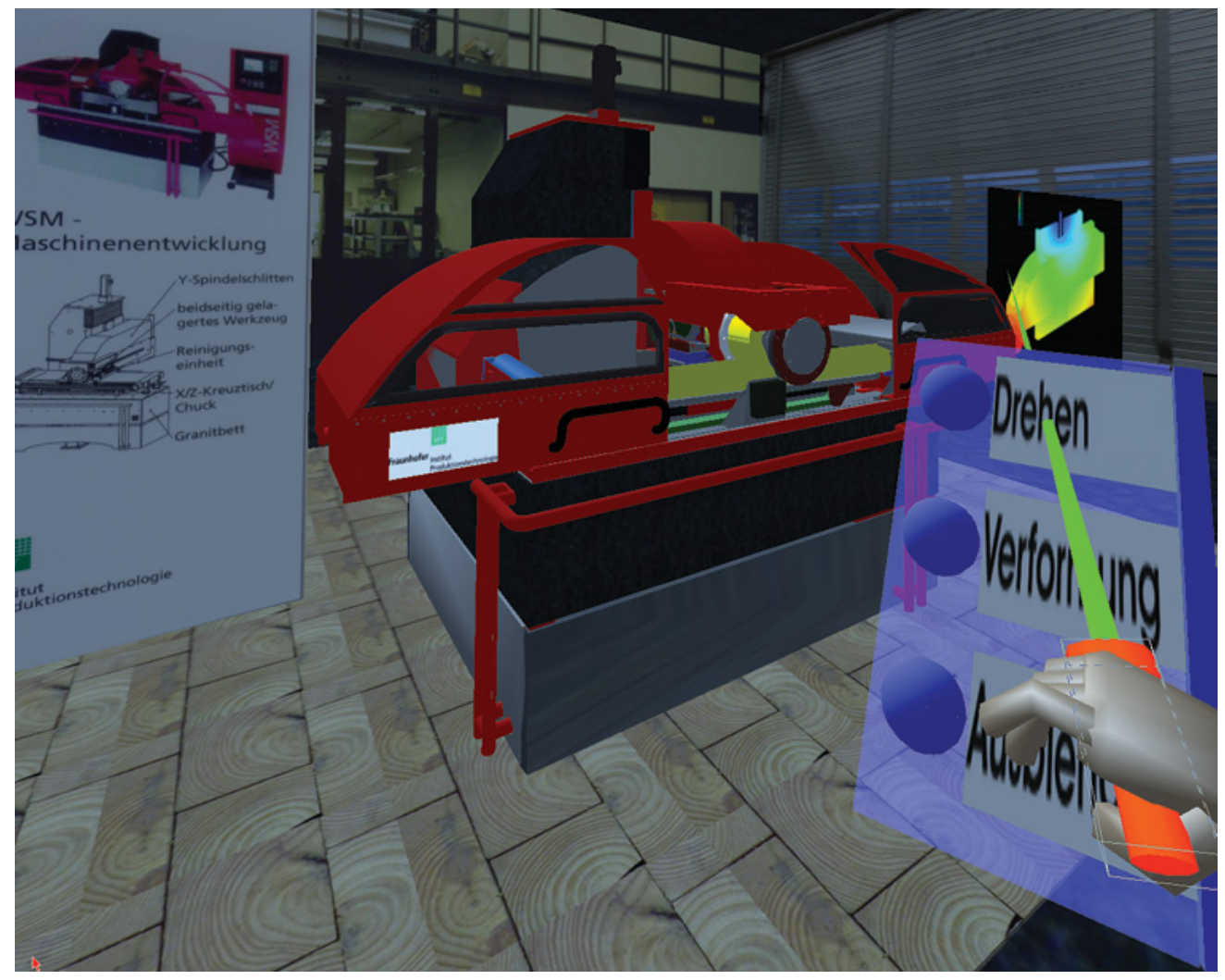

Fig. 1. Digital Mock-Up of a wafer grinding machine in a virtual environment.

Their video information will then be transferred via optical network to your VR system so that you can see and interact with your partners, and vice versa. The virtual objects will also be shared so that collaborative sessions will be possible.

The area of process planning is currently not supported by VR. The proposal to use Virtual Reality for the post processing of FEM data of forming processes is expressed by Roll et al. [7]. The main benefit of VR post processing compared to conventional post processing lies in the reduction of evaluation time. This decrease is due to the use of multiprocessors and multipipe hardware architectures. Furthermore, the stereovisualisation of complex three-dimensional processes permits a better understanding of the processes, for example surface structure of simulated machined workpieces is easier to observe. A third reason to develop VR for process planning is the intuitive interaction using tracked input devices. In this way, it is possible to simply cut the workpiece in the virtual 3D environment and place cutting planes without having to enter coordinates. For a detailed evaluation of different model versions, it is also possible to enter coordinates. Covise/Cover was mainly developed for the post processing of computational flow data (CFD), and therefore, further development of the software is necessary to adapt to the evaluation of manufacturing processes.

\section{An approach to integrate VR, FEM, and simulation tools}

\subsection{Examples of process, tool, machine tool, and control system integration}

In the manufacturing chain, there are several areas where integration of VR, FEM, and other simulation tools is necessary. Four application areas can be identified: the process, the tool, the machine tool, and the control system.

For an integrated process simulation, it is necessary to take the material history, or, in other words, the prior processing steps, into account. A good example is the simulation of a car crash. A crash simulation relies upon the material properties of all parts involved. All parts are assumed to behave ideally. Sheet thickness variations and strain hardening in the material are not currently considered in crash simulations. Because of the use of ideal properties, there can be changes in the stiffness of the car up to ten percent in comparison between simulated and actual results according to Roll et al. [7]. In contrast, an integrated process would start from the sheet metal forming simulation that imparts a variation in sheet thickness and a permanent strain to the parts. These results could then be imported into the crash simulation and 
consequently lead to a more realistic model. In addition, the results of the crash simulation could be used to selectively influence the sheet metal forming process of the parts to increase the car body stiffness.

An example of an integrated simulation between a process and a tool is a forging process. Today, forging processes are primarily derived assuming ideal behavior, which means that no elastic deformation of the tool can occur. In reality, the elastic deformation of the tool deforms the workpiece from its ideal geometry. The integrated process would consist of a combined simulation where the forging process is computed elastically and plastically while the tool is only modelled elastically. Examples of such a coupled simulation without the use of VR for post processing are described in [11]. The process forces are used to calculate the elastic deformation of the tool.

To integrate one step further, the machine tool has to be taken into account. The integrated simulation would start, for example, with the simulation of a cutting process. The forces and heat from cutting are transferred to the tool, which then deforms elastically. The forces, temperature, and elastic deformation of the tool affect the structure of the entire machine tool, which can also elastically deform. This then results in a displacement of the cutting tool, which consequently affects the cutting process. An example of a coupled forging simulation can be found in [16]. Until now, this type of simulation has been done without the aid of VR.

As the final element, the control system must be included to monitor the whole system in an integrated simulation where simulation tools from the process, the tool, the machine tool, and the control system are coupled. The control unit has to interpret the numerical code, feed the virtual machine axis, and guide the tool and that way influence the process.

\subsection{Methods of coupling simulations}

In considering the integrated simulations described above, there are three different ways to couple the simulations: loose, tied, and retroactive.

In a loose coupling, separate simulations for the process and the tool are computed, and the results between the simulations are then transferred manually in one direction. This method could be used to calculate the elastic deformation of a tool according to the process forces at a certain time step.

In a tied coupling, separate simulations for the process and the tool are computed, and the results of the process simulation are then transferred through a specially designed interface to the tool simulation for each time step. This method allows for the analysis of elastic deformation of the tool over the whole manufacturing process and identifies critical process steps.

Retroactive coupling for simulations most accurately represents reality. This coupling includes the tied coupling data exchange and further incorporates the retroactive influence of both simulation processes.
Wiemer has studied the coupling of forming processes [17].

\subsection{Realised integration of VR and FEM in the manufacturing process chain}

In our first implementation, a drilling process and drill has been integrated with VR post processing provided by Covise [13]. The process data consisted of the FEM mesh and stress and strain values for each element. The process was computed with Deform3D at the Laboratory for Machine Tools and Production Engineering. An interface has been programmed that read the ASCII output from Deform3D and translated it into the data structure of Covise. The FEM model consisted of 37598 tetrahedron elements and 310 time steps, with each time step $0.8 \mathrm{~ms}$ and each element width 100 microns. The chip formation is shown in Figure 2. In the virtual environment, it is possible to observe the bending of the chips. Furthermore, it is possible to enlarge the object so that inside an individual chip, cutting planes can be positioned to observe the gradients of stress, strain, or temperature from the surface to the middle of the chip. With a probe tool, it is possible to select discrete points so that the $x-, y-$, and $z$ coordinates and their corresponding values are displayed.

Secondly, the interface has been extended to also read the drill geometry. The drill was modelled in the CAD system IDEAS and transferred via the STL-interface (Standard Triangulation Language) to Deform3D. The cutting edge had to be highly detailed for the FEM analysis. The drill was modelled as a rigid body in the FEM system and consisted of 12628 polygons. The detailed mesh of the drill made interaction difficult, because of a decreased frame rate. To overcome this issue, initially every second polygon was skipped during the conversion with our interface. Afterwards, interaction was possible again, but the missing polygons led to holes in the visualised drill. Instead, a simplifying algorithm was used provided by Covise in addition to a module that allows parts of the drill to be removed from the model. Cutting the drill in half then made realtime interaction possible without the holes from the first solution. Figure 2 shows the VR post processing with the process, the drill, and the evaluation menus.

\section{Problems to solve}

In "Requirements for the simulation of the system machine - tool - process", Großmann [18] describes three different views of an integrated simulation environment: the object view, the user view, and the data view.

The object view takes into account all components or parts that are involved in the simulation: the manufacturing process, the parts of the machine tool, and the control system. In this area, problems with the simulation of objects must be solved, such as the integration of elastically deformable tools in the process simulation. New evaluation tools for VR post processing must also be developed 


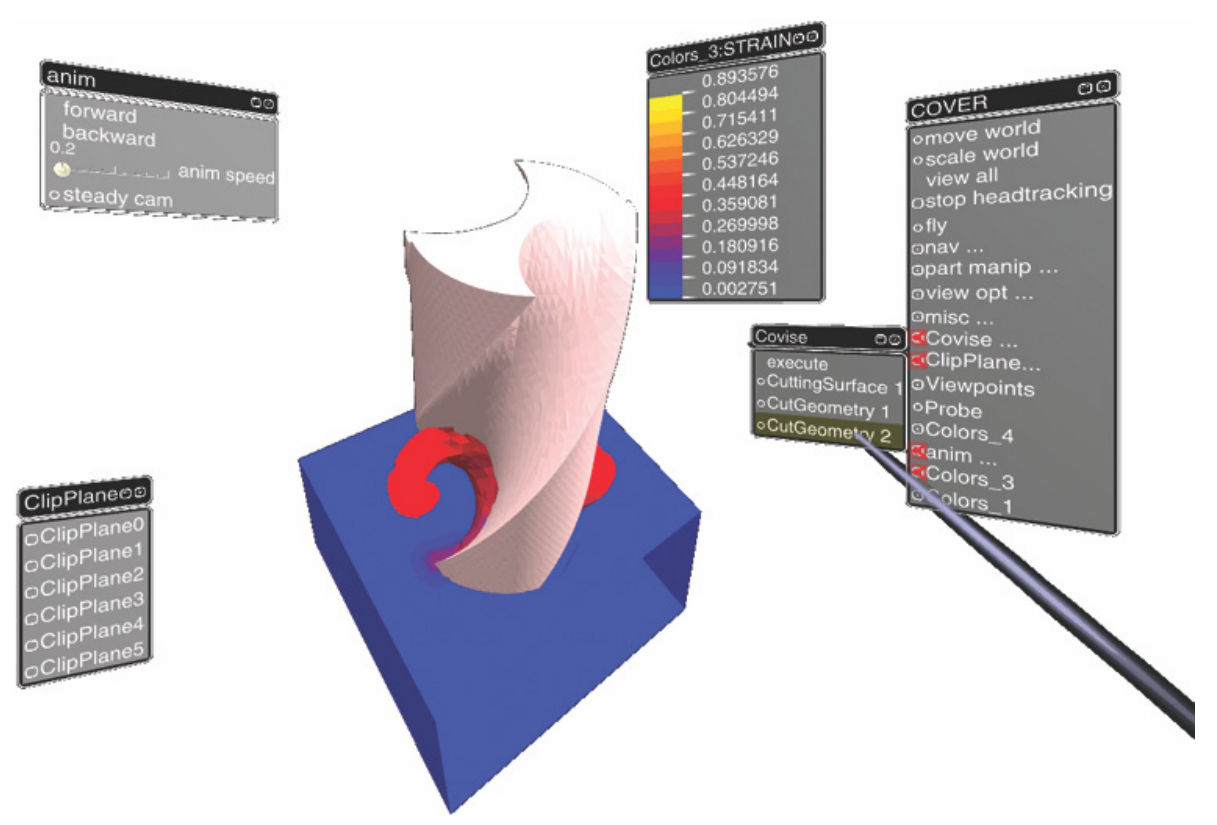

Fig. 2. VR post processing of FEM computed drilling process.

which support the combined visualisation of objects from different simulation tools.

The user needs a system with intuitive controls and easy acclimation, clear symbols and terms, service and support, a practical parameter dialogue, and interfaces for data exchange. The application of VR can assist in meeting these needs. But the accessibility of VR is limited by the high initial investment. Present efforts to incorporate $\mathrm{PC}$ clusters with consumer graphics cards are helping to lower the initial investment. An increasing number of VR systems run under Linux, and further functionality is constantly being added. Along with this development, administration of PC clusters will become a more important issue.

The majority of problems must be solved in the data area. First, the strength of existing CAD and FEM systems must be harnessed. FEM systems commonly use different element representations in models. Depending on the solver algorithm, hexahedron or tetrahedron elements, for example, may be used. To couple the simulation results from different FEM systems, it is necessary to develop interpolation programs that match the resulting values from one simulation model to another [7]. Each CAx system focuses on solving a specific problem and thus provides unique solutions, which when combined can benefit the manufacturing chain. Therefore, interfaces between all involved systems must be designed, such as the interface implemented for Deform3D. These interfaces should not only work in one direction but also enable the transfer of results into the previous step. The user could review the simulation results, find an undesired manufacturing process characteristic, and then wish to make a change. Instead of going back to a separate program, it should be possible to change the tool dimensions, for example, directly in VR. These changes should automatically be reflected in the FEM simulation that then starts calculating with the new parameters. The user would receive an estimate for the calculation time and could decide when to schedule the next meeting. These interfaces will require a great deal of work, but in the end, the user will be rewarded with greater productivity. The user will be able to use his experience and familiarity with particular software and can then use the designed interface to transfer his results to another person specialised in a different piece of simulation software. A system that can perform all simulation steps necessary to solve every problem in a virtual manufacturing process is unlikely to exist or to be developed. The need for individual interfaces between FEM systems is also mentioned in [19].

Computation time for simulations is currently a limiting factor. Depending on the model size and other simulation parameters, such as time steps, computations can vary from hours to days. The continuing development of processing units and the improvement of solver algorithms will undoubtedly reduce computation times.

Different simulation times limit the coupling of simulations. Two different methods of coupling can be identified based on simulation time: asynchronous and realtime coupling. The application of VR ideally warrants realtime coupling, which unfortunately cannot be achieved with the simulation times of modern FEM processes. In our research, only the application of asynchronous coupling is possible. With asynchronous coupling, the simulation results are stored in a database. The database can also be used for version control of the simulation results. Dierssen performed a coupling of a multi-body simulation with the simulation of an operating sequence and visualisation with a VR system [20]. In a similar approach the computation time for the multi-body simulation took over two hours whereas the time for the logistic simulation was on the order of seconds [19]. 


\section{Conclusions}

It could be demonstrated that it is possible to utilize the VR system Covise to perform VR post processing of the simulation results provided by the FEM software Deform3D. In this study, an interface has been programmed to exchange data from a $3 \mathrm{D}$ drilling process. It was possible to visualise 310 steps with each of the 37598 nodes in an animation. During this animation, the geometry could be cut, and cutting planes and isosurfaces could be interactively placed. Furthermore, discrete values of points could be requested through the interactive placement of a probe tool. In the next step, the interface was enhanced to import the drill tool. Strategies to reduce the number of polygons of the drill were applied to successfully achieve realtime interaction.

In the future, the drill will be modelled elastically so that the torsion of the drill is accounted for. Additional evaluation tools will be developed to analyse the progression of the cutting force during the process. Furthermore, it should be possible to change simulation input parameters during VR post processing. This will trigger new FEM calculations and generate new results that are then reincorporated in the $\mathrm{VR}$ post processing.

The integration of VR, FEM, and other simulation tools will combine the benefits from all tools involved. With respect to the simulation tools, this work will enhance the understanding of manufacturing processes down to the level of physical transactions and allow for the evaluation of different scenarios to determine the influence of each parameter. With respect to Virtual Reality, the advantages of better team communication, combined visualisation of simulation results, and the VR benefits of stereo visualisation, intuitive navigation and evaluation, and realtime interactions will be realized.

Acknowledgements. The authors wish to thank the BMBF Bundesministerium für Bildung, Wissenschaft, Forschung und Technologie (German Federal Ministry of Education, Science, Research and Technology) and their industrial partners for funding parts of the work presented.

\section{References}

[1] D. Clausing, Total Quality Development - A Stepby-Step Guide to World-Class Concurrent Engineering, ASME Press, 1994

[2] S. Nahavandi, C. Preece, A Virtual Manufacturing Environment with an Element of Reality, in: Proceedings of the Fourth International Conference on Advanced Factory Automation, UK, 1994, pp. 624-629

[3] M. Hitchcock, A. Baker, J. Brink, The Role of Hybrid Systems Theory in Virtual Manufacturing, in: Proceedings of the IEEE Conference, 1994, pp. 345-350
[4] C. Shukla, M. Vazquez, F. Chen, Virtual Manufacturing: An Overview, in: International Conference on Computers and Industrial Engineering 31(1), 1996, pp. 79-82

[5] G. Mukundan, Editorial Virtual Manufacturing, in: Journal of Advanced Manufacturing Systems 1, 2002, pp. 1-3

[6] N.H. Robertson, T. Perera, Feasibility for Automatic Data Collection, in: Proceedings of the 2001 Winter Simulation Conference, Arlington, 2001, pp. 984-990

[7] K. Roll, T. Altan, A.E. Tekkaya, M. Hermann, Virtuelle Umformtechnik, in: Umformtechnik 2000 PLUS, Meisenbach Bamberg, 1999, pp. 255-274

[8] F. Klocke, Th. Beck, St. Hoppe., T. Krieg, N. Müller, T. Nöthe, H.W. Raedt, K. Sweeney, Examples of FEM application in manufacturing technology, in: Journal of Materials Processing Technology 120, 2002, pp. 450-457

[9] S.V.G. Cobb, M.D. D'Cruz, J.R. Wilson, Integrated Manufacturing: A role for virtual reality?, in: International Journal of Industrial Ergonomics 16, 1995, pp. 411-425

[10] F. Klocke, A.M. Straube, G. Messner, T. Bergs, Virtual Process Engineering, submitted in response to Call EOI. FP6. 2002 of the European Commision, 2002

[11] C. Caesar, H. Flegel, D. Friedrich, J. Grams, P.O. Hahn, F. Klocke, D. Lung, S. Nöken, D. Pähler, W. Schmidt, G. Trommer, K. Weinert, H. Willms, R. Zeller, Design and Optimisation of Process Chains - Today's and Tomorrow's Implementation of Alternative Production Sequences, in: AWK2002, Aachen, 2002

[12] D. Friedrich, C. Gottschalk, H. G. Haldenwanger, F. Klocke, R. Kopp, D. Lung, D. Löhe, L. Markworth, A. Ostertag, H. W. Raedt, K. Steffens, U. Wunsch, P. Zeller, Simulation of Manufacturing Processes - Potentials and Limits of Part Design, in: AWK2002, Aachen, 2002

[13] R. Lang, D. Rainer, U. Woessner, P. Wolf, VirCinity, Covise Module Reference Guide, 2001

[14] vrcom GmbH, virtual design 2 User Guide, 2000

[15] I. Nikitin, L. Nikitina, P. Frolov, G. Goebbels, M. Goebel, S. Klimenko, G.M. Nielson, Real-time simulation of elastic objects in Virtual Environments using finite element methods and precomputed Green's functions, in: Proc. of EGVE 2002, Barcelona, 2002, p. 47

[16] K. Großmann, Zukunftspotential - Simulation, in SIM2000, Dresden, 2000, pp. 1-23

[17] F. Schirmacher, H. Wiemer, Kopplung von Prozess- und Anlagensimulation - ein wesentlicher Schritt auf dem Weg zum, Virtuellen Umformen, in: EFB-Kolloquium, Stuttgart, 2001, pp. 11.1-11.18

[18] K. Großmann, H. Wiemer, Anforderungen an die Simulation des Systems, Maschine-Werkzeug-Prozess in der Umformtechnik, in SIM2000, Dresden, 2000, pp. $775-793$

[19] F. Christoph, T. Collisi, R. Kohlbauer, R. Völkl, C. Effert, T. Oertli, T. Siggenauer, Durchgängiger Einsatz der Simulationstechnik, in VDI-Z, 144, No. 3, 2002

[20] S. Dierssen, Die Virtuelle Maschine: Konfiguration, Simulation, Visualisierung, in: Simulation und Visualisierung 2000, Magdeburg, 2000 